\title{
Memory cost for simulating all quantum correlations from the Peres-Mermin scenario
}

\author{
Gabriel Fagundes ${ }^{1,2, *}$ and Matthias Kleinmann ${ }^{2}$ \\ ${ }^{1}$ Departamento de Física, Universidade Federal de Minas Gerais UFMG, \\ P.O. Box 702, 30123-970, Belo Horizonte, MG, Brazil \\ ${ }^{2}$ Department of Theoretical Physics, University of the Basque Country UPV/EHU, P.O. Box 644, 48080 Bilbao, Spain
}

\begin{abstract}
Sequences of compatible quantum measurements can be contextual and any simulation with a classical model conforming with the quantum predictions needs to use internal memory. Kleinmann et al. [New J. Phys. 13, 113011 (2011)] showed that simulating the sequences from the Peres-Mermin scenario requires at least three different internal states in order to be not in contradiction with the deterministic predictions of quantum theory. We extend this analysis to the probabilistic quantum predictions and ask how much memory is required to simulate the correlations generated for sequences of compatible observables by any quantum state. We find that even in this comprehensive approach only three internal states are required for a perfect simulation of the quantum correlations in the Peres-Mermin scenario.
\end{abstract}

\section{INTRODUCTION}

In the standard formulation of quantum theory (QT) the individual outcomes of measurements are, in general, not predetermined by the state of the system. Consequently, QT allows us to asses only the probability distribution over the measurement outcomes. Specker [1] noted that this is a fundamental property of QT and if quantum measurements had predetermined outcomes it would imply that these values depend on the measurement context. In this sense, QT is contextual and the mathematical formulation of this observation is the Kochen-Specker theorem [2].

Significant effort has been undertaken to understand the connection between quantum contextuality and quantum information theory, for example, with respect to the advantage of quantum computing over classical computing $[3,4]$. Similarly, a quantum system distributed over several parties can be used to reduce the communication complexity over what is possible with classical systems alone $[5,6]$ and the communication advantage has been identified as a resource [7-9]. A related concept is the memory cost in sequential measurements $[10,11]$, i.e., the memory needed to simulate the correlations occurring in sequences of quantum measurements by means of a classical automaton with memory. It has been found that the memory cost can exceed the amount of information that can be stored in the quantum system yielding a quantum memory advantage [10-12]. We are here interested in the analysis of the memory cost with respect to quantum contextuality, i.e., to determine the memory cost when the measurements in a sequence only embraces mutually compatible measurements [10]. In this strict form the question of whether there exists a quantum memory advantage due to contextuality is still open.

In this paper we investigate the situation for one of the most natural candidates for a quantum memory advantage, the Peres-Mermin square. We ask, what is

* gabrielf@fisica.ufmg.br the smallest memory for a classical model to reproduce all contextuality predictions from the Peres-Mermin scenario, for any quantum state. Our focus here is to stay strictly in the regime of quantum contextuality, i.e., sequences of compatible measurements, and to take into account also the probabilistic predictions of quantum theory, while at the same time to admit the most versatile classical automaton models.

\section{THE PERES-MERMIN SQUARE}

A simple proof of the Kochen-Specker theorem was found by Peres [13] and Mermin [14] and uses 9 quantum observables arranged in the Peres-Mermin square,

$$
\left[\begin{array}{ccc}
A & B & C \\
a & b & c \\
\alpha & \beta & \gamma
\end{array}\right]=\left[\begin{array}{ccc}
\sigma_{z} \otimes \mathbb{1} & \mathbb{1} \otimes \sigma_{z} & \sigma_{z} \otimes \sigma_{z} \\
\mathbb{1} \otimes \sigma_{x} & \sigma_{x} \otimes \mathbb{1} & \sigma_{x} \otimes \sigma_{x} \\
\sigma_{z} \otimes \sigma_{x} & \sigma_{x} \otimes \sigma_{z} & \sigma_{y} \otimes \sigma_{y}
\end{array}\right],
$$

where $\sigma_{x}, \sigma_{y}$, and $\sigma_{z}$ are the Pauli operators. The proof of the theorem consists of the observations (i) that the operators within each row and each column form a context, i.e., they are mutually compatible, and (ii) that the condition

$$
A B C=a b c=\alpha \beta \gamma=A a \alpha=B b \beta=-C c \gamma=\mathbb{1}
$$

holds. Therefore, according to QT, the expected value of the product of the outcomes of observables in one context is always +1 , with the exception $\langle C c \gamma\rangle=-1$. In order to obtain this behavior if the values of the observables are predetermined, at least one observable needs to have a context-dependent value, so that, for example, $\gamma$ has value +1 in the context $\alpha \beta \gamma$ but value -1 in the context $C c \gamma$.

In QT, the outcomes of all observables within a context can be obtained in a joint measurement. For the three dichotomic observables in each context of the Peres-Mermin square, the joint measurement on two qubits has four distinct outcomes, taken from the set of the 8 possible combinations of outcomes 
$\{(+1,+1,+1),(+1,+1,-1), \ldots,(-1,-1,-1)\}$. Alternatively, the outcomes can be obtained by measuring the observables in a context sequentially. This approach has been preferred in recent experiments on quantum contextuality [15-20]. When an observable $X$ from the Peres-Mermin square is measured, then the quantum state $\rho$ changes according to

$$
\rho \mapsto \frac{\Pi_{x \mid X} \rho \Pi_{x \mid X}}{\operatorname{tr}\left(\rho \Pi_{x \mid X}\right)},
$$

with $\Pi_{x \mid X}=\frac{1}{2}(\mathbb{1}+x X)$ depending on the measurement outcome $x= \pm 1$ of $X$. In a sense, sequential measurements with this Lüders transformation [21] are a special way to implement a joint measurement. Since the quantum state changes according to the choice of the observable and the measurement outcome, one can argue that the quantum state serves as a memory and the contextual behavior is achieved due to the very presence of this memory.

However, in an extended variant of the Peres-Mermin square, it has been shown [10] that even if one takes this perspective, a classical model mimicking the quantum behavior would need more than four internal states. This extended scenario uses quantum predictions for all combinations of Pauli matrices on two qubits, resulting in 15 dichotomic observables. The classical model must then reproduce the predictions from any sequence of compatible observables as well as respect conditions of compatibility and repeatability. The latter include conditions on sequences of incompatible measurements, and thus are outside the contextuality paradigm. Since the extended variant also operates on a quantum four-level system and such systems can carry at most two bits of classical information [22], this has been identified as an instance of memory advantage [10-12].

The analysis in Ref. [10] concerns classical models which reproduce the deterministic predictions of QT within a sequence. Such predictions are, for example, that the product of outcomes in the sequence $A, B, C$ is always +1 or that the value of $A$ is repeated in the sequence $A, B, A$. For the case of the Peres-Mermin square and when any sequence of measurements consists of observables from one context, there is a classical model consistent with QT in this sense and which only uses three internal states. This analysis does not cover the probabilistic predictions of $\mathrm{QT}$, for example, that $\langle A\rangle=0$ for certain quantum states and it is not known how much memory is needed to reproduce also the probabilistic predictions of QT in the Peres-Mermin square. Since the Peres-Mermin scenario is tightly linked to contextuality, we only consider sequences of observables taken from one context. This includes predictions like $\langle B B A\rangle=\langle A\rangle$, but excludes predictions involving incompatible observables as in $\langle A B c \gamma\rangle=-1$. In this paper our aim is hence to determine the smallest memory for a classical model to reproduce the nondeterministic contextual quantum predictions from the Peres-Mermin scenario, for any quantum state.

\section{SEQUENTIAL CORRELATIONS AND STOCHASTIC AUTOMATA}

The outcomes of a sequence of quantum measurements may be viewed as an input-output process operating on a quantum system. The input is the choice of the observable $X$ and the output is the outcome $x$ of the measurement of the observable. The overall probability for an output sequence $x_{1}, x_{2}, \ldots$ for a given input sequence $X_{1}, X_{2}, \ldots$ is $P\left(x_{1}, x_{2}, \ldots \mid X_{1}, X_{2}, \ldots\right)$ and within standard QT only such correlations can be predicted.

The classical counterpart is modeled by an automaton which operates on classical memory. This memory is represented by a set $M$ of internal memory states. In addition, the automaton has access to an external source of randomness, modeled by an external parameter $\lambda$ which is fixed throughout a measurement sequence but randomly distributed among different sequences according to a distribution function $p(\lambda)$. We use the model of a stochastic sequential automaton [23] where the output $x$ and the state $s^{\prime} \in M$ after the output only depend on the input $X$, the value of $\lambda$, and the internal state $s \in M$ before the output, cf. Fig 1 . The behavior of the automaton is hence summarized by the probability distribution $p\left(x, s^{\prime} \mid X, s, \lambda\right)$. It represents the probability of the output $x$ and subsequent transition to the internal state $s^{\prime}$, given the input $X$, the current internal state $s$ and the value of the parameter $\lambda$. Similarly, the initial state of the automaton has a distribution depending on $\lambda$, which we write as $p\left(s_{0} \mid \lambda\right)$. With this model, the correlations achieved by the automaton are

$$
\begin{aligned}
& P\left(x_{1}, x_{2}, \ldots \mid X_{1}, X_{2}, \ldots\right)= \\
& \sum_{\lambda, s_{0}, s_{1}, s_{2}, \ldots} p(\lambda) p\left(s_{0} \mid \lambda\right) p\left(x_{1}, s_{1} \mid X_{1}, s_{0}, \lambda\right) \\
& \times p\left(x_{2}, s_{2} \mid X_{2}, s_{1}, \lambda\right) \cdots
\end{aligned}
$$

For a given automaton, i.e., $p\left(x, s^{\prime} \mid X, s, \lambda\right)$ and $p\left(s_{0} \mid \lambda\right)$, the choice of $p(\lambda)$ yields different correlations, so that the correlations predicted by different quantum states can be reproduced using different choices of the probability distribution $p(\lambda)$.

Clearly, it is possible to reproduce all noncontextual correlations with only one internal state, $|M|=1$, since in this case the right hand side of Eq. (4) reduces to a hidden variable model [24], $\sum_{\lambda} p(\lambda) p\left(x_{1} \mid X_{1}, \lambda\right) p\left(x_{2} \mid X_{2}, \lambda\right) \cdots$. The external parameter $\lambda$ is not always included in such an analysis, see, for example, the $\epsilon$-transducers studied in Ref. [25]. However, then even noncontextual scenarios could require memory, since, for example, measuring the sequence $\sigma_{x}, \sigma_{x}$ on an eigenstate of $\sigma_{z}$ gives a random outcome for the first measurement, but the second measurement has to repeat the value of the first measurement. Consequently, if $\lambda$ does not occur, the simulation requires two internal states, while when $\lambda$ can take two values, no memory is required. The automaton is allowed to be intrinsically random, i.e., the distributions $p\left(x, s^{\prime} \mid X, s, \lambda\right)$ and 


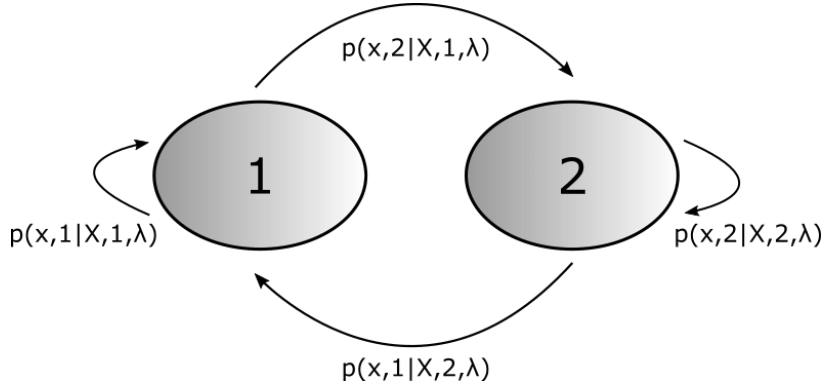

FIG. 1. Stochastic sequential machine with two internal states, $M=\{1,2\}$. The transitions between the states $s$ and $s^{\prime}$ are represented by arrows. The probability $p\left(x, s^{\prime} \mid X, s, \lambda\right)$ for the output $x$ and the transition from state $s$ to state $s^{\prime}$ can depend on the input $X$ and the external parameter $\lambda$.

$p\left(s_{0} \mid \lambda\right)$ may be nondeterministic. As it is evident from our analysis below, this intrinsic randomness is not required for simulating the quantum correlations from the Peres-Mermin scenario.

\section{A MEMORY-OPTIMAL AUTOMATON FOR THE PERES-MERMIN SCENARIO}

As explained above, quantum contextuality is a feature of sets of compatible observables and we therefore only consider sequences of measurements where all observables are taken from one context. That is, the observables are either taken from one row or one column of the Peres-Mermin square. Our first concern is the simulation of quantum measurements of a single sequence of compatible observables. According to QT, certain events can never occur, examples are the output $+1,-1$ in the sequence $A, A$ or the output $+1,+1,-1$ in the sequence $A, B, C$. In Ref. [10], it has been shown that any automaton which obeys all such quantum predictions must have memory with at least three internal states. An explicit example of such an automaton is given by [10]

$$
\begin{aligned}
& o_{1}=\left[\begin{array}{lll}
+1 & +1 & +1 \\
+1 & +1 & +1 \\
+1 & +1 & +1
\end{array}\right], \quad t_{1}=\left[\begin{array}{lll}
1 & 1 & 2 \\
1 & 1 & 3 \\
1 & 1 & 1
\end{array}\right], \\
& o_{2}=\left[\begin{array}{lll}
+1 & +1 & +1 \\
-1 & +1 & -1 \\
-1 & -1 & +1
\end{array}\right], \quad t_{2}=\left[\begin{array}{lll}
2 & 1 & 2 \\
2 & 2 & 2 \\
2 & 3 & 2
\end{array}\right], \\
& o_{3}=\left[\begin{array}{lll}
+1 & -1 & -1 \\
+1 & +1 & +1 \\
-1 & -1 & +1
\end{array}\right], t_{3}=\left[\begin{array}{lll}
3 & 3 & 3 \\
1 & 3 & 3 \\
2 & 3 & 3
\end{array}\right] .
\end{aligned}
$$

This notation is supposed to be read as follows. Each matrix $o_{i}, i \in M$, represents the deterministic output for each of the three internal states $M=\{1,2,3\}$. Similarly, the transition matrices $t_{i}$ represent the internal state after the output. In terms of Eq. (4), the distribution $p\left(x, s^{\prime} \mid X, s\right)$ is 1 if the entry in the output matrix $o_{s}$ at the position of the observable $X$ is $x$ and the entry in the transition matrix $t_{s}$ in the same position is $s^{\prime}$; the distribution is 0 otherwise. Here, $x \in\{+1,-1\}$, $s, s^{\prime} \in M=\{1,2,3\}$, and $X \in\{A, B, C, a, b, c, \alpha, \beta, \gamma\}$. For example, if the automaton is in state $s=1$ and we provide the observable $C$ as input, then the measurement outcome is $x=+1$ and the automaton changes to the state $s^{\prime}=2$. It is straightforward to verify that this automaton obeys all deterministic predictions of QT for any sequence of compatible observables [10] and for any initial state $s_{0}$.

However, no quantum state gives deterministic predictions for all 9 observables in the Peres-Mermin square, because these observables are not all mutually compatible and no common eigenstate can exist. In the following we extend the automaton from Eq. (5) to use the external parameter $\lambda$, so that a statistical mixture $p(\lambda)$ can reproduce the quantum predictions.

\section{A. Other valid automata}

Starting from the automaton in Eq. (5), there are several transformations which lead to other automata with the same properties. First, it is possible to flip the signs for the output under the constraint that for each row and each column there is either no flip of signs or there are exactly two flips of signs. This generates 15 additional automata. Second, it is possible to make any permutation of the rows or a permutation of the first and second column. We restrict ourselves to the three permutations of rows which leave one row unchanged and to the permutation of the first and second column. This yields 4 additional automata and combined with the first set of transformations we get in total $16 \times 5=80$ automata. In addition, we are free to choose the initial state $s_{0}$ and get this way 240 different behaviors. We combine all these behaviors into a single automaton by allowing 240 different values for $\lambda$, i.e., the value of $\lambda$ determines the behavior of the automaton.

\section{B. Example: Singlet state}

As an example, we reproduce all quantum correlations for the singlet state (the quantum state yielding $\langle C\rangle=$ $\langle c\rangle=\langle\gamma\rangle=-1$ ) by choosing a distribution $p(\lambda)$ for $\lambda=$ $1,2, \ldots, 240$. We choose $p(\lambda)=\frac{1}{4}$ if $\lambda \in\left\{\lambda_{1}, \lambda_{2}, \lambda_{3}, \lambda_{4}\right\}$ and $p(\lambda)=0$ else. For $\lambda_{k}, k=1,2,3$, the transition matrices $t_{1}^{(k)}, t_{2}^{(k)}, t_{3}^{(k)}$ are as in Eq. (5) and the outcome matrices $o_{s}^{(k)}$ are given by

$$
\begin{array}{r}
o_{1}^{(1)}=\left[\begin{array}{lll}
-1 & +1 & -1 \\
-1 & -1 & +1 \\
+1 & -1 & -1
\end{array}\right], o_{2}^{(1)}=\left[\begin{array}{lll}
-1 & +1 & -1 \\
+1 & -1 & -1 \\
-1 & +1 & -1
\end{array}\right], \\
o_{3}^{(1)}=\left[\begin{array}{lll}
-1 & -1 & +1 \\
-1 & -1 & +1 \\
-1 & +1 & -1
\end{array}\right],
\end{array}
$$




$$
\begin{array}{r}
o_{1}^{(2)}=\left[\begin{array}{lll}
-1 & +1 & -1 \\
+1 & +1 & +1 \\
-1 & +1 & -1
\end{array}\right], o_{2}^{(2)}=\left[\begin{array}{lll}
-1 & +1 & -1 \\
-1 & +1 & -1 \\
+1 & -1 & -1
\end{array}\right], \\
o_{3}^{(2)}=\left[\begin{array}{lll}
-1 & -1 & +1 \\
+1 & +1 & +1 \\
+1 & -1 & -1
\end{array}\right],
\end{array}
$$

$$
\begin{array}{r}
o_{1}^{(3)}=\left[\begin{array}{lll}
+1 & -1 & -1 \\
-1 & -1 & +1 \\
-1 & +1 & -1
\end{array}\right], o_{2}^{(3)}=\left[\begin{array}{lll}
+1 & -1 & -1 \\
+1 & -1 & -1 \\
+1 & -1 & -1
\end{array}\right], \\
o_{3}^{(3)}=\left[\begin{array}{lll}
+1 & +1 & +1 \\
-1 & -1 & +1 \\
+1 & -1 & -1
\end{array}\right],
\end{array}
$$

$$
\begin{array}{r}
o_{1}^{(4)}=\left[\begin{array}{lll}
+1 & -1 & -1 \\
+1 & +1 & +1 \\
+1 & -1 & -1
\end{array}\right], o_{2}^{(4)}=\left[\begin{array}{lll}
+1 & -1 & -1 \\
-1 & +1 & -1 \\
-1 & +1 & -1
\end{array}\right] \\
o_{3}^{(4)}=\left[\begin{array}{lll}
+1 & +1 & +1 \\
+1 & +1 & +1 \\
-1 & +1 & -1
\end{array}\right] .
\end{array}
$$

The initial state for all four cases is $s_{0}=2$, i.e., we have $p\left(s_{0} \mid \lambda\right)=1$ if $s_{0}=2$ and zero else. In principle one can now verify that for sequences of compatible observables, all quantum correlations from the singlet state are indeed reproduced. However, there is an infinite number of input sequences which needs to be considered and it is our next step to reduce the number of sequences to a finite set.

\section{A finite set of sufficient input sequences}

We show in this section that a finite number of input sequences suffices to determine all correlations for all sequences. Since we only consider sequences of observables from one context, as soon as two different observables occur in a sequence, it is already possible to predict the reminder of the sequence from the outcome of these two observables. This is because the product of outcomes of the three observables of each context is always +1 or -1 , depending on the context, and due to the requirement that repeated occurrences of an observable in a sequence produce repeated values.

Hence, it remains to consider sequences where initially one observable is measured repeatedly, for example, $X, X, Y$. In quantum mechanics we have

$$
\begin{aligned}
& P(x, x, \ldots, x, y \mid X, X, \ldots, X, Y)= \\
& \operatorname{tr}\left(\Pi_{y \mid Y} \Pi_{x \mid X} \rho \Pi_{x \mid X} \Pi_{y \mid Y}\right)=P(x, y \mid X, Y),
\end{aligned}
$$

for any number of repetitions of the input $X$ and output $x$. However, for the automaton model we could have different values for outcome $y$ in the sequences $X, X, \ldots, X, Y$, depending on the number of repetitions of $x$, since the value of $Y$ does not need to be fixed until $Y$ is actually measured. Thus, we have to consider how our specific model behaves in this situation. For any value of $\lambda$, the behavior of our automaton is analogous to the automaton in Eq. (5) and for this automaton one observes that the internal state $s^{\prime}$ after an $\ell$-fold measurement of $X$ does not depend on $\ell$, if $\ell \geq 1$. Hence, the outcome of $X, X, \ldots, X, Y$ is $x, x, \ldots, x, y$ if and only if $X, Y$ has outcome $x, y$.

In summary, our automaton with any choice of $p(\lambda)$ reproduces the quantum correlations for a state $\rho$ for all sequences of compatible observables, if and only if it does so for all sequences of length two. For practical reasons, instead of dealing with the correlations $P(x, y \mid X, Y)$ we use the equivalent set of expectation values

$$
\begin{aligned}
\langle X\rangle & =\sum_{x, y} x P(x, y \mid X, Y), \\
\langle X Y X\rangle & =\sum_{x, y} y P(x, y \mid X, Y), \text { and } \\
\langle X Y\rangle & =\sum_{x, y} x y P(x, y \mid X, Y),
\end{aligned}
$$

where in the second equation we used that the value of $X$ in the first and in the last position are the same. Note, that while in $\mathrm{QT}$, we always have $\langle X Y X\rangle=\langle Y\rangle$, this does not hold for all ensembles $p(\lambda)$ in our automaton, as, for example, in Eq. (5) with initial internal state $s=1$, we have $\langle c\rangle=1$, but $\langle C c C\rangle=-1$. However, we observe that $\langle X Y\rangle=\langle X Y\rangle$ for all $p(\lambda)$ and all compatible $X$ and $Y$, a relation that also holds in QT for any state.

Therefore, we have to take into account 9 values $\langle X\rangle$, 18 values $\langle X Y\rangle$, and 36 values $\langle X Y X\rangle$. We enumerate these values by $j=1, \ldots, 63$ and collect for each $j$ the values for all 240 values of $\lambda$ in a vector $\vec{v}_{j}$. Then the expectation values $\vec{q}=\left(q_{1}, \ldots, q_{63}\right)$ can be achieved if and only if $q_{j}=\vec{v}_{j} \cdot \vec{p}$ for some probabilities $\vec{p}$ with $p_{\lambda} \equiv$ $p(\lambda)$. The set of achievable expectation values $\vec{q}$ is hence given by the polytope

$$
P=\left\{\vec{q} \mid q_{j}=\vec{v}_{j} \cdot \vec{p} \text { for all } j \text { and some } \vec{p}\right\} .
$$

Similarly, for the quantum correlations we have 63 hermitian operators $Z_{j}$, such that the expectation values $\vec{q}$ can be attained according to QT if and only if $q_{j}=\operatorname{tr}\left(\rho Z_{j}\right)$ for all $j$ and some quantum state $\rho$. The set of achievable expectation values $\vec{q}$ according to QT is consequently the convex set

$$
Q=\left\{\vec{q} \mid q_{j}=\operatorname{tr}\left(\rho Z_{j}\right) \text { for all } j \text { and some } \rho\right\} .
$$

This allows us to easily verify the correctness of the example in Sec. IV B, by comparing $\vec{v}_{j} \cdot \vec{p}$ with $\operatorname{tr}\left(\rho Z_{j}\right)$ for all $j$ and for any quantum state $\rho$, finding a corresponding distribution $p(\lambda)$ reduces to find probabilities $\vec{p}$ with $\vec{v}_{j}$. $\vec{p}=\operatorname{tr}\left(\rho Z_{j}\right)$ for all $j$. This can be solved by means of linear programming and was in fact our method to find $p(\lambda)$ for the singlet state in Sec. IV B. 


\section{Simulation of the correlations of any quantum state}

We are now equipped with the necessary tools to prove that the correlations of any quantum state can be simulated with a construction analogous to the one in Sec. IV B. According to our previous analysis, the question whether the quantum predictions can be simulated by an appropriate distribution $p(\lambda)$ reduces to the question whether the convex set $Q$ is contained in the polytope $P$. In order to make this question tractable, we use an equivalent representation of the polytope, where it is written as a finite intersection of half-spaces [26] parametrized by vectors $\vec{h}_{\ell}$ and numbers $\alpha_{\ell}$, so that

$$
P=\left\{\vec{q} \mid \vec{h}_{\ell} \cdot \vec{q} \leq \alpha_{\ell} \text { for all } \ell\right\}
$$

Using this half-space representation, $P$ contains $Q$ if and only if $\vec{h}_{\ell} \cdot \operatorname{tr}(\rho \vec{Z}) \leq \alpha_{\ell}$ for all $\ell$ and all $\rho$. By writing

$$
W_{\ell}=\alpha_{\ell} \mathbb{1}-\vec{h}_{\ell} \cdot \vec{Z}
$$

this further simplifies to $\operatorname{tr}\left(\rho W_{\ell}\right) \geq 0$ for all $\ell$ and all $\rho$. That is, $Q \subset P$ holds if and only if all $W_{\ell}$ are positive semidefinite. Conversely, if we find a state with $\operatorname{tr}\left(\rho W_{\ell}\right)<0$ for some $\ell$, and hence $W_{\ell}$ is not positive semidefinite, then our automaton cannot simulate all quantum predictions for this state.

In principle, this can be tested directly. However, since the polytope $P$ is given in the form of Eq. (9), we need to compute the half-space representation in Eq. (11). This can be achieved by using the Fourier-Motzkin elimination, but is known to be a computationally hard task and for our problem we were not able to find a direct solution. The central observation to solve the problem nonetheless is that $Q$ spans a rather low-dimensional affine space. In particular, $Q$ is contained in the affine space $\vec{a}+U \equiv\{\vec{a}+\vec{u} \mid \vec{u} \in U\}$, where $a_{j}=\operatorname{tr}\left(\rho Z_{j}\right)$ for some fixed $\rho_{0}$ (for example, $\rho_{0}=\frac{1}{4} \mathbb{1}$ ) and $U$ is the linear space $U=\left\{\vec{u} \mid u_{j}=\operatorname{tr}\left(G Z_{j}\right)\right.$ for some $\left.G\right\}$ with $G$ any hermitian operator obeying $\operatorname{tr}\left(\rho_{0} G\right)=0$. This holds true since we can always write $\rho=\rho_{0}+G$ for some $G$. The dimension of the linear space $U$ is only $\operatorname{dim} U=9$, as can be found by using the linear independence relations of the operators $Z_{j}$. Therefore, $Q \subset P$ is equivalent to $Q \subset P \cap(\vec{a}+U)$ and our problem reduces to calculate a half-space representation for the polytope $P \cap(\vec{a}+U)$. This problem is easily tractable, as we discuss in Appendix A. We obtain 24 nonzero operators $W_{\ell}$, each of which is positive semidefinite. This proves $Q \subset P$ and thus our automaton can simulate the quantum correlations for any quantum state. We mention that the nonzero operators $W_{\ell}$ are, up to an arbitrary positive factor, exactly those 24 projectors of unit rank which commute with all observables from one out of the six contexts in the Peres-Mermin square.

\section{CONCLUSIONS}

Quantum contextuality is considered as one of the key differences between the microscopic world and the world governed by classical mechanics. Recent experimental demonstrations of this phenomenon proceed by measuring sequences of observables and yield a contradiction to the assumption of noncontextuality, i.e., the assumption that the value of an observable does not depend of which other compatible observables are measured alongside. We revisited this conclusion for the case of the Peres-Mermin scenario in the light of classical models which utilize internal memory in order to reproduce the quantum behavior. We showed that for this scenario an automaton using only three internal states can reproduce the quantum correlations from any quantum state for any sequence of compatible observables. This model is also optimal, since a lower bound of three internal states was already established [10]. The memory cost of the Peres-Mermin scenario is therefore actually lower than the canonical quantum implementation, which requires two qubits. Since for quantum correlations involving sequences of incompatible observables, the memory cost can also be larger than the memory of the quantum system, this leaves open the question, whether there can be a quantum memory advantage when restricted to sequences of compatible observables and if so, for which contextuality scenario this occurs.

\section{ACKNOWLEDGMENTS}

We thank Costantino Budroni, Adán Cabello, Marcelo Terra Cunha, Jan-Åke Larsson, Marco Túlio Quintino, and Géza Tóth, for discussions. This work was supported by CNPq, Conselho Nacional de Desenvolvimento Científico e Tecnológico, Brazil, the FQXi Large Grant "The Observer Observed: A Bayesian Route to the Reconstruction of Quantum Theory", the EU (ERC Starting Grant GEDENTQOPT), and by the DFG (Forschungsstipendium KL 2726/2-1).

\section{Appendix A: Low-dimensional section of a polyhedral cone}

A central step in Sec. IV D is to compute the half-space representation of the polytope $P \cap(\vec{a}+U)$, where $P$ is a polytope, $\vec{a} \in P$ is a vector and $U$ is a linear subspace of low dimension.

We first consider the equivalent problem for a polyhedral cone $\mathcal{P}=\{A \vec{r} \mid \vec{r} \succeq 0\}$, where $\vec{r} \succeq 0$ abbreviates $r_{k} \geq 0$ for all $k$ and $A$ is some matrix with real entries. For a matrix $K$, let $F$ be a matrix the range of which is 
the kernel of $K A$. We have

$$
\begin{aligned}
\mathcal{P} \cap \operatorname{ker}(K) & =\{A \vec{r} \mid \vec{r} \succeq 0, K(A \vec{r})=0\} \\
& =\{A \vec{r} \mid \vec{r} \succeq 0, \vec{r}=F \vec{s} \text { for some } \vec{s}\} \\
& =\{A F \vec{s} \mid F \vec{s} \succeq 0\} \\
& =A F\{\vec{s} \mid F \vec{s} \succeq 0\},
\end{aligned}
$$

where we used that $K A \vec{r}=0$ implies $\vec{r}=F \vec{s}$ for some $\vec{s}$ and, conversely, $(K A) F \vec{s}=0$ for any $\vec{s}$. It follows that if we can obtain a matrix $F^{\prime}$, such that $\{\vec{s} \mid F \vec{s} \succeq 0\}=$ $\left\{F^{\prime} \vec{s} \mid \vec{s} \succeq 0\right\}$, then $\mathcal{P} \cap \operatorname{ker}(K)=\left\{A F F^{\prime} \vec{s} \mid \vec{s} \succeq 0\right\}$.

For our case, we extend the polytope $P$ from Eq. (9) to a polyhedral cone $\mathcal{P}$ by adding $\vec{e}=(1,1, \ldots, 1)$ to the vectors $\vec{v}_{j}$ and by dropping the constraint $\sum_{i} p_{i}=$ 1, i.e., $\mathcal{P}=\{A \vec{r} \mid \vec{r} \succeq 0\}$ and $A$ is the matrix with rows $\left[e, v_{1}, \ldots, v_{63}\right]$. Then $(1, \vec{q}) \in \mathcal{P}$ if and only if $\vec{q} \in P$. Similarly, we define the linear subspace $\mathcal{U}=$ $\{(\lambda, \lambda \vec{a}+\vec{u}) \mid \lambda \in \mathbb{R}$ and $\vec{u} \in U\}$, so that $(1, \vec{x}) \in \mathcal{U}$ is equivalent to $\vec{x} \in \vec{a}+U$.

In order to apply Eq. (A1), we choose some matrix $K$ such that $\operatorname{ker}(K)=\mathcal{U}$ and some matrix $F$ with range
$\operatorname{ker}(K A)$. Despite $F^{T}$ being a larger matrix than $A$, we find that $F^{\prime}$ is rather easy to compute. The ma$\operatorname{trix} B=A F F^{\prime}$ is then only of $\operatorname{rank} \operatorname{dim}(\mathcal{U})=10$ and a matrix $B^{\prime}$ with $\{B \vec{s} \mid \vec{s} \succeq 0\}=\left\{\vec{y} \mid B^{\prime} \vec{y} \succeq 0\right\}$ can be computed at an instance. We use the software cddlib [27] to generate the matrices $F^{\prime}$ and $B^{\prime}$ and iml [28] to compute $K$ and $F$. Both packages work with unlimited exact integer arithmetic and hence our computation of $B^{\prime}$ is exact. We verify independently our results by using porta [29] to compute $K, F, F^{\prime}$ and $B^{\prime}$.

Finally, we have that $\vec{q} \in P$ and $\vec{q} \in \vec{a}+U$ if and only if $(1, \vec{q}) \in \mathcal{P} \cap \mathcal{U}$, i.e., if and only if $B_{\ell, 1}^{\prime}+\sum_{j} B_{\ell, j+1}^{\prime} q_{j} \geq 0$ for all $\ell$. Therefore, the operators $W_{\ell}$ defined in Eq. (12) are given by

$$
W_{\ell}=B_{\ell, 1}^{\prime} \mathbb{1}-\sum_{j} B_{\ell, j+1}^{\prime} Z_{j}
$$

As we showed in the main text, $Q \subset P \cap(\vec{a}+U)$ is equivalent to all $W_{\ell}$ being positive semidefinite. In our analysis, all operators $W_{\ell}$ satisfy this condition.
[1] Ernst Specker, "Die Logik nicht gleichzeitig entscheidbarer Aussagen," Dialectica 14, 239-246 (1960).

[2] Simon Kochen and Ernst P. Specker, "The problem of hidden variables in quantum mechanics," J. Math. Mech. 17, 59-87 (1967).

[3] Robert Raussendorf, "Contextuality in measurementbased quantum computation," Phys. Rev. A 88, 022322 (2013).

[4] Mark Howard, Joel Wallman, Victor Veitch, and Joseph Emerson, "Contextuality supplies the 'magic' for quantum computation," Nature (London) 510, 351-355 (2014).

[5] B. F. Toner and D. Bacon, "Communication cost of simulating Bell correlations," Phys. Rev. Lett. 91, 187904 (2003).

[6] Harry Buhrman, Richard Cleve, Serge Massar, and Ronald de Wolf, "Nonlocality and communication complexity," Rev. Mod. Phys. 82, 665-698 (2010).

[7] Rodrigo Gallego, Lars Erik Würflinger, Antonio Acín, and Miguel Navascués, "Operational framework for nonlocality," Phys. Rev. Lett. 109, 070401 (2012).

[8] Julio I de Vicente, "On nonlocality as a resource theory and nonlocality measures," J. Phys. A: Math. Theo. 47, 424017 (2014).

[9] Jonathan Barrett, Noah Linden, Serge Massar, Stefano Pironio, Sandu Popescu, and David Roberts, "Nonlocal correlations as an information-theoretic resource," Phys. Rev. A 71, 022101 (2005).

[10] Matthias Kleinmann, Otfried Gühne, José R. Portillo, Jan-Åke Larsson, and Adán Cabello, "Memory cost of quantum contextuality," New J. Phys. 13, 113011 (2011).

[11] Stephen Brierley, Adrian Kosowski, Marcin Markiewicz, Tomasz Paterek, and Anna Przysiężna, "Nonclassicality of temporal correlations," Phys. Rev. Lett. 115, 120404 (2015).

[12] Ernesto F. Galvão and Lucien Hardy, "Substituting a qubit for an arbitrarily large number of classical bits," Phys. Rev. Lett. 90, 087902 (2003).

[13] Asher Peres, "Incompatible results of quantum measurements," Phys. Lett. A 151, 107-108 (1990).

[14] N. David Mermin, "Simple unified form for the major nohidden-variables theorems," Phys. Rev. Lett. 65, 33733376 (1990).

[15] G. Kirchmair, F. Zähringer, R. Gerritsma, M. Kleinmann, O. Gühne, A. Cabello, R. Blatt, and C. F. Roos, "State-independent experimental test of quantum contextuality," Nature (London) 460, 494-497 (2009).

[16] Elias Amselem, Magnus Rådmark, Mohamed Bourennane, and Adán Cabello, "State-independent quantum contextuality with single photons," Phys. Rev. Lett. 103, 160405 (2009).

[17] Radek Łapkiewicz, Peizhe Li, Christoph Schaeff, Nathan K. Langford, Sven Ramelow, Marcin Wieśniak, and Anton Zeilinger, "Experimental non-classicality of an indivisible quantum system," Nature (London) $\mathbf{4 7 4}$, 490-493 (2011).

[18] Xiang Zhang, Mark Um, Junhua Zhang, Shuoming An, Ye Wang, Dong-ling Deng, Chao Shen, Lu-Ming Duan, and Kihwan Kim, "State-independent experimental test of quantum contextuality with a single trapped ion," Phys. Rev. Lett. 110, 070401 (2013).

[19] Vincenzo D'Ambrosio, Isabelle Herbauts, Elias Amselem, Eleonora Nagali, Mohamed Bourennane, Fabio Sciarrino, and Adán Cabello, "Experimental implementation of a Kochen-Specker set of quantum tests," Phys. Rev. X 3, 011012 (2013).

[20] Markus Jerger, Yarema Reshitnyk, Markus Oppliger, Anton Potočnik, Mintu Mondal, Andreas Wallraff, Kenneth Goodenough, Stephanie Wehner, Kristinn Juliusson, Nathan K. Langford, and Arkady Fedorov, "Contextuality without nonlocality in a superconducting quantum system," Nat. Commun. 7, 12930 (2016). 
[21] Gerhart Lüders, "Über die Zustandsänderung durch den Meßprozeß," Ann. Phys. (Leipzig) 443, 322-328 (1951).

[22] A. S. Holevo, "Bounds for the quantity of information transmitted by a quantum communication channel," Probl. Inf. Transm. 9, 177-183 (1973).

[23] Azaria Paz, Introduction to Probabilistic Automata (Academic Press, New York, London, 1971).

[24] J. S. Bell, "On the Einstein Podolsky Rosen paradox," Physics 1, 195-200 (1964).

[25] Nix Barnett and James P. Crutchfield, "Computational mechanics of input-output processes: Structured transformations and the $\epsilon$-transducer," J. Stat. Phys. 161,
404-451 (2015).

[26] Branko Grünbaum, Convex polytopes, 2nd ed. (Springer, New York, 2003).

[27] K. Fukuda, "cddlib v094h," https://www.inf.ethz.ch/ personal/fukudak/cdd_home/.

[28] Z. Chen, A. Storjohann, and C. Fletcher, "Integer Matrix Library v1.0.5," https://cs.uwaterloo.ca/ astorjoh/ iml.html.

[29] T. Christof and A. Löbel, "POlyhedron Representation Transformation Algorithm v1.4.1," http://porta.zib. $\mathrm{de} /$. 